\title{
Design and Development of Light Radiation Testing Equipment for Solar Greenhouse
}

\author{
Yang Wenxiong ${ }^{1, *}$, Ma Chengwei ${ }^{2}$ \\ ${ }^{1}$ Beijing Vocational College of Agriculture, Beijing 102242, China \\ ${ }^{2}$ China Agricultural University, Beijing 100083, China
}

\begin{abstract}
A solar greenhouse light radiation testing equipment is designed and developed. The equipment consists of five parts: light radiation sensor, angle adjustment module, height adjustment module, data acquisition box and base module. It is easy to move, easy to use, detachable and assembleable, and can accurately obtain the illumination of the solar greenhouse everywhere, so as to help farmers use sunlight scientifically and rationally, precisely plant, and play a role in increasing production and efficiency. The equipment is not available on the market at present and belongs to original design.
\end{abstract}

\section{Introduction}

Solar greenhouse accounts for more than $60 \%$ of China's facility agriculture, but the regulation and control of its environmental factors basically depend on the experience of technical personnel, especially the lack of understanding of indoor light distribution in solar greenhouse, which leads to farmers' failure to make full and reasonable use of sunlight, resulting in the reduction of greenhouse output and insignificant economic benefits [1]. Therefore, accurate acquisition and scientific analysis of the illuminance of light radiation in all parts of the greenhouse are essential to effectively improve the efficiency of light utilization and increase production and income [2-5].

However, at present, the light radiation testing equipment is mainly used outside the greenhouse, which is highly fixed and bulky, so it is impossible to accurately obtain the light radiation illuminance in all parts of the greenhouse. As a result, when farmers are planting greenhouse crops, they cannot make full and reasonable use of the sunlight, resulting in a reduction of more than $10 \%$ in the production of greenhouse crops. The economic benefits and enthusiasm of farmers are not significant, even some villages and towns abandon the solar greenhouse. The extensive use of light energy production mode deviates from the development requirements of precision agriculture in China [6-8].

In order to solve this problem, this paper designs and develops a kind of solar greenhouse light radiation testing equipment with independent intellectual property rights, which is convenient to move, easy to use, detachable and installable, low cost, and can accurately obtain the light radiation illuminance of all parts of the solar greenhouse, so as to help farmers scientifically and reasonably use the sunlight, accurately plant, and play the role of increasing production and efficiency [9].

\section{Structure and composition}

The solar greenhouse light radiation test equipment is a kind of special equipment for measuring the light radiation illuminance in the solar greenhouse, with a height of $0.4-2 \mathrm{~m}$. It is composed of five parts: light radiation sensor, angle adjustment component, height adjustment component, data acquisition box and base component. The overall assembly of the equipment is shown in Fig. 1, and the working state after assembly is shown in Fig. 2 and Fig. 3.

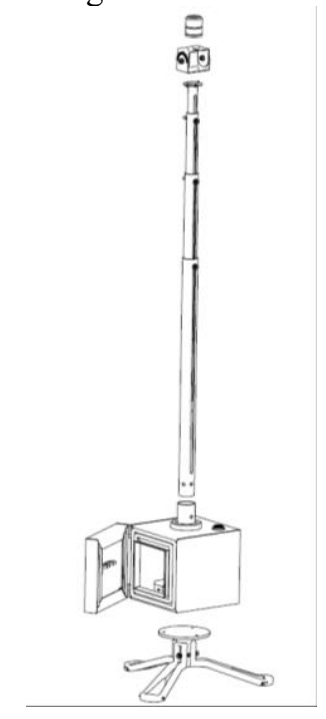

Figure 1 Assembly drawing of solar greenhouse light radiation testing equipment

\footnotetext{
* Corresponding author: 24518518@qq.com
} 


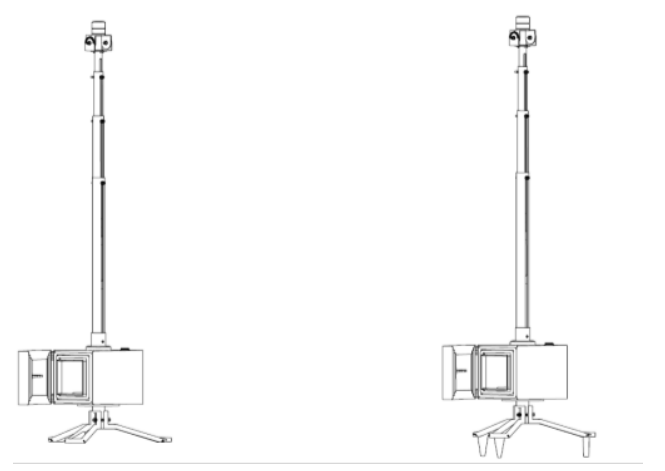

Figure 2 Vertical state, Supporting condition of solar greenhouse light radiation testing equipment under different ground

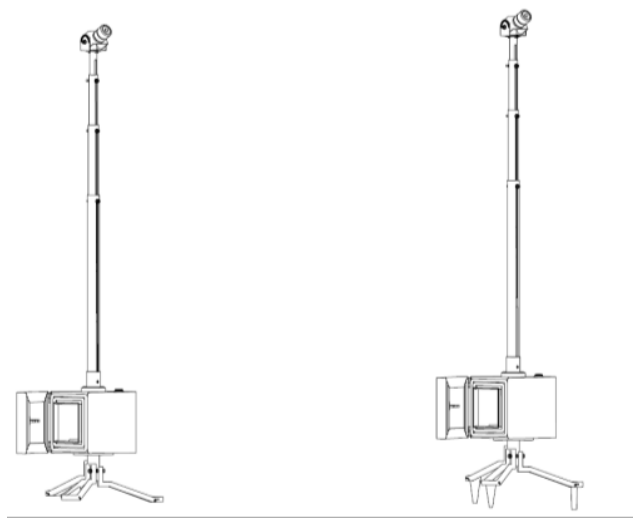

Figure 3 Inclined state, Supporting condition of solar greenhouse light radiation testing equipment under different ground

\section{Connection and use}

The light radiation sensor is located on the top of the whole equipment to ensure the accurate collection of light radiation illuminance in the solar greenhouse without shielding around. The bottom end and the plane above the angle adjusting assembly are fixed with nuts.

The angle adjusting assembly is composed of upper part, lower part and two screws, as shown in Figure 4. There are two circular through holes on the left and right sides of the upper part and the lower part. The two parts are connected by two screws. When we adjust the angle, we loosen the screw to adjust the relative angle of the two parts, and then tighten the screw, so that the angle of the radiator sensor changes with respect to the ground. The upper part is marked with zero position mark, and the lower part is marked with scale. By aligning the mark and scale, the required angle can be indicated. The adjustment range of the angle is $-90^{\circ}-+90^{\circ}$. The two nuts of the upper part are welded on the body to facilitate screw tightening and loosening, and the nuts will not be lost. The upper part of the angle adjusting assembly is provided with two circular holes for connecting with the holes of the optical radiometer sensor, and a small Ushaped wire through hole for passing through the data line of the optical radiation sensor.

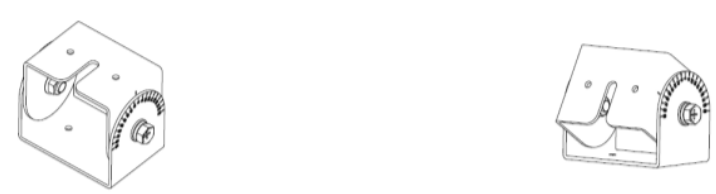

Figure 4 Angle adjustment component

The height adjusting part is composed of the top bar, the adjustable bar, the adjustable bar and the bottom bar, as shown in Figure 5. There is a platform above the top bar, and there are two circular holes on the platform, which are used to connect with the angle adjusting component. There are also small U-shaped wire holes on the top bar. If the angle does not need to be adjusted, the light radiation sensor can also be directly fixed on the top bar. The top bar, adjustable bar and bottom bar are hollow round bars, of which 2-3 adjustable bars can be installed as required. Each rod has a long circular adjusting hole. By changing the relative position of each rod, the different height of the light radiation sensor in the solar greenhouse can be adjusted steplessly.

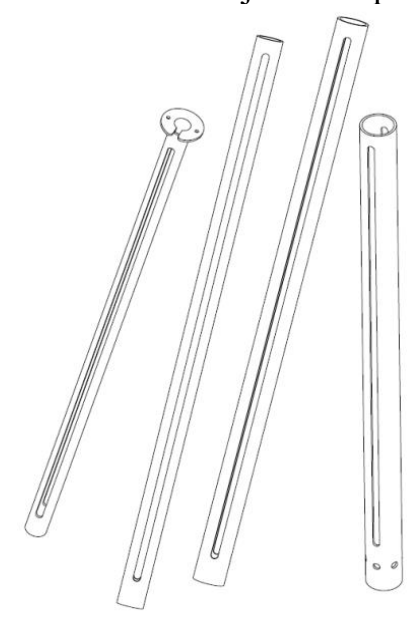

Figure5 Regulator rod assemblies

The data acquisition box is located directly below the height adjusting rod, and its central axis is coaxial with the height adjusting rod to ensure the stability of the whole equipment, as shown in Fig. 6. The data acquisition box is composed of box, box cover, upper part of box, base of level meter, ring level meter, waterproof plug, tool support, door lock, data collector and waterproof sealing ring. The upper part of the box body is a round pipe, which is welded on the box body. The bottom rod of the height adjusting component is inserted into it and fixed by screws. The door lock is installed on the lid to prevent theft. There are two semicircular grooves on the box body and the box cover, and two waterproof sealing rings are bonded in the grooves. When the box cover is closed, the whole data collection box is waterproof to ensure that the data collector is in a dry environment. The box cover is also equipped with a tool bracket, which is convenient for the placement of the screws used in the installation and disassembly of the equipment and other tools, so as to prevent the loss. The box body is also equipped with a level base, which is in the shape of a circular ring and is provided with a 
semicircle groove for placing the level in the shape of a circular ring. By observing the position of the air bar inside the circular level, the whole box body is adjusted to keep horizontal. The box is equipped with a waterproof plug, which is used to connect the data line between the light radiation sensor and the data collector to ensure the strong waterproof of the whole collection. The data collector consists of three parts: the collector box, the collector cover and the collector circuit board. All these elaborate designs are to ensure that the data acquisition box can work normally in the solar greenhouse environment with high temperature and humidity for a long time.

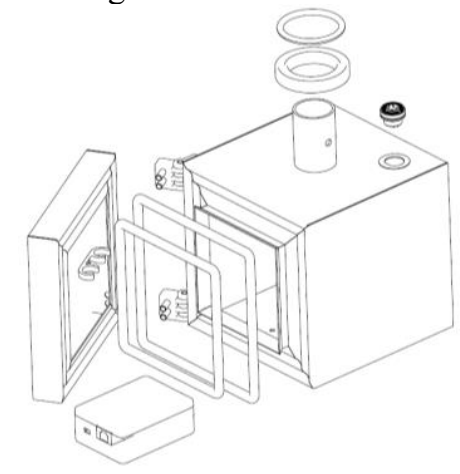

Figure 6 Explosion chart of data acquisition box

The base assembly is located at the bottom of the entire device and is connected to the bottom of the data acquisition box by three screws, as shown in Figure 7 . The base assembly is composed of an inverted T-shaped column, three base supporting rods and a foot rotating part. The substructure strut can be adjusted up and down along the inverted $\mathrm{T}$-shaped column. If the ground is flat, the three substructure struts are in the same horizontal plane; if the ground is uneven, the position of the three substructure struts in the vertical direction can be adjusted to ensure that the upper plane of the inverted Tshaped column is horizontal. There are two shafts on the side of the rotating part of the support foot, which can rotate along the support rod of the base. There are two positioning holes 1 and 2 on both sides of the support rod of the base. When the floor is a concrete floor or brick surface, the rotating part of the support foot is located at a position parallel to the support rod of the base, and the screw is inserted from the positioning hole 1 to fix the rotating part of the support foot at position 1 . When the ground is clay ground, we can rotate the rotating part of the support foot to the vertical state, insert the screw from the positioning hole 2, so as to fix the rotating part of the support foot at the position 2, so that the rotating part of the support foot can be inserted into the clay ground to ensure the stability of the whole device. By adjusting the base support rod and the rotating part of the support foot, the base component can adapt to the flat ground, the uneven ground, the hard ground on the concrete ground or brick surface, and the soft ground such as soil.

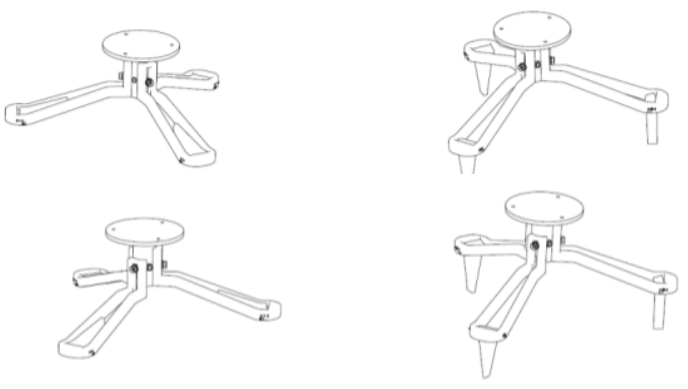

Figure 7 Four states of base adjusting assembly

\section{Features and application}

The test equipment is a special equipment for measuring the light radiation in the solar greenhouse. At present, there is no such equipment on the market, which belongs to the original design.

The height of the light radiation sensor of the test equipment can be adjusted steplessly, so that the light radiation value at different height can be measured conveniently. The adjusting hole of the height adjusting rod is oblong and can be adjusted to any height within a certain range.

The angle of the light radiation sensor of the test equipment can be adjusted, so that the light radiation values of different angles at the same height can be easily measured. The adjustment range is $-900-+900$. The angle adjusting component is marked with scale, which can accurately adjust the angle of the radiometer.

The angle adjusting assembly has a small U-shaped gap, which is convenient for the data line of the optical radiation sensor to pass through, without removing the line and reinstalling it.

The test equipment can adapt to various complex terrain. It can not only adapt to flat ground, but also adapt to convex-concave ground. It can not only adapt to the hard ground of concrete or brick surface, but also adapt to the soft ground such as soil. This is very important for the situation that the measuring points need to be changed frequently in the solar greenhouse.

The center of gravity of the data acquisition box of the test equipment is located on the central axis of the whole equipment and at the bottom of the equipment, which reduces the center of gravity of the system and makes the equipment have good stability. This can make the height adjusting rod very thin, make the bottom bracket as small as possible, so that the whole equipment is light and flexible, and easy to move frequently. Generally, similar devices and data boxes are hung on the side of the vertical bar, so the vertical bar is thick and strong to prevent toppling, which will cause the equipment to be heavy and inconvenient to move.

The data collection box of the test equipment is sealed by two layers of waterproof sealing rings, and the data line is connected by waterproof plug to ensure good sealing effect and extend the service life of the data collector.

There is a tool support behind the door of the data acquisition box of the test equipment, which is convenient to place the screwdriver and other tools used 
in the installation or removal of the device, so as to prevent the loss.

There is a bracket above the data acquisition box of the test equipment for placing the ring level, which is convenient for adjusting the whole equipment to ensure that it is in a state perpendicular to the ground.

There is a circular platform on the top bar of the height adjustment component, and there are also small U-shaped through-hole and two circular through-hole. If it is not necessary to adjust the angle, the optical radiation sensor can be directly fixed on the top bar.

The light radiation sensor is located on the top of the whole equipment and will not be affected by the light shielding of the equipment itself.

The parts of the equipment can be disassembled to facilitate transportation and movement.

\section{Conclusion}

Through equipment development and experiment test, the solar greenhouse light radiation test equipment can improve the light utilization efficiency of the solar greenhouse by $5-10 \%$, and the corresponding crop output by $4-8 \%$. According to the annual average minimum income of each solar greenhouse of 60000 yuan RMB, the annual economic benefit of a single solar greenhouse can be increased by $2400-4800$ yuan RMB. According to the average of 30 solar greenhouses owned by agricultural facility enterprises, it is expected that the annual economic benefit can be increased by 30 solar greenhouses The economic income increased by 72000 144000 yuan RMB and the effect of increasing production and income was significant.

\section{References}

1. P. Peng, L.Liang, H. Li, Nor Horticul. 5, 161(2019)

2. Q .Gao, Y. Liang, A.Duan, Trans. Chin. Soc. Agric. Eng. 19, 200(2003)

3. J .Qu, H. Xu, R .Wang, J. Northwest Sci-Tech Univ. Agric. For.39,178(2011)

4. J. Wu, Y.Xie, B. An, Shandong Agric. Sci. 45,118 (2013)

5. H. Liu, W. Guo, H.Li, J Meteor Res 19, 350 (2008)

6. G. Tong, B. Li , J. China Agric. Univ. 11, 61 (2006)

7. X.Li, J. Heilongjiang Sci-Tech Univ. 15, 27 (2005)

8. C. Ma, S. Zhao, J. Cheng, J. Shenyang Agric. Univ.44, 513 (2013)

9. W. Yang, K.Ye, J. Li, J. Anhui Agric. Sci. 26,180 (2018) 